\title{
Research on Camouflage Recognition in Simulated Operational Environment Based on Hyperspectral Imaging Technology
}

\author{
Donge Zhao $\mathbb{i D}^{1,2}$ Shuyan Liu ${ }^{10},{ }^{1}$ Xuefeng Yang, ${ }^{1}$ Yayun $\mathrm{Ma}^{1}{ }^{1}$ Bin $\mathrm{Zhang}^{1}$ and Wenbo Chu ${ }^{1}$ \\ ${ }^{1}$ School of Information and Communication Engineering, North University of China, Taiyuan 030051, China \\ ${ }^{2}$ Shanxi Provincial Research Center for Opto-Electronic Information \& Instrument Engineering Technology, \\ North University of China, Taiyuan 030051, China \\ Correspondence should be addressed to Donge Zhao; zhaodonge@nuc.edu.cn
}

Received 5 January 2021; Revised 31 March 2021; Accepted 1 April 2021; Published 14 April 2021

Academic Editor: Khalique Ahmed

Copyright ( $\odot 2021$ Donge Zhao et al. This is an open access article distributed under the Creative Commons Attribution License, which permits unrestricted use, distribution, and reproduction in any medium, provided the original work is properly cited.

\begin{abstract}
Hyperspectral imaging technology can obtain the spatial information and spectral information of the simulated operational background and its camouflage materials at the same time and identify and classify them according to their differences. In this paper, we collected the hyperspectral images $(400-1000 \mathrm{~nm})$ of the desert background, jungle background, desert camouflage netting, jungle camouflage netting, and jungle camouflage clothing through the hyperspectral imaging system, and the samples were preprocessed by denoising and black-and-white correction. Then, we analysed the region of interest (ROI) of the training samples by principal component analysis (PCA). After the pixels in the region of interest and their surrounding areas were averaged, $60 \%$ of the data was used as the training samples, and the remaining $40 \%$ was used as the test samples. According to their similarities and differences between them and referenced spectrum, the models of classification were established by combining the Naive Bayes (NB) algorithm, K-nearest neighbour (KNN) algorithm, random forest (RF) algorithm, and support vector machine (SVM) algorithm. The results show that among the four models, SVM model has the highest accuracy of classification and the recognition rate of jungle camouflage clothing is the highest. This study verifies the scientific and feasibility of hyperspectral imaging technology for camouflage identification and classification in a simulated operational environment, which has some practical significance.
\end{abstract}

\section{Introduction}

Camouflage technology uses patterns close to the background to achieve the purpose of hiding, which can not only enhance the concealment of the target but also improve the survivability of the target on the combat environment. Traditional detection and recognition of camouflage targets mostly relied on magnifying observations of human eyes by optical instruments, but the effect of detection was usually poor [1]. Nowadays, intelligence and informatization have become the main forms of detection and identification in the modern confrontational environment [2]. How to deal with the high-tech reconnaissance in the scene of confrontation has become an important aspect of military technology, resulting in the emergence of many high-tech disguises.

In recent years, the multiband camouflage netting produced by Swedish Barracuda Company has adopted the principle of new absorption and ultralight structure and achieved the purpose of multispectral stealth compatibility through a multifunctional coating technology [3]. The ultralight camouflage netting system produced in the USA consists of hexagonal and diamond shapes joined by loops of rope, which are light and stable for use in camouflage netting system for infrared [4]. A neopentyl glycol system was prepared and optimised in China. Polyester hollow fibers are filled by the vacuum method and woven into camouflage clothing [5]. With the diversification of camouflage technology in combat environment, it is difficult to evaluate the stealthy performance and survivability of targets comprehensively and accurately by traditional methods.

Hyperspectral imaging technology can record the spatial information and spectral information of the targets [6]. By introducing it into the evaluation system of camouflage effectiveness, the difference between the target and the 
background in high-dimensional space can be used to detect the target, which will greatly improve the detection efficiency and ability of camouflage target [7]. Consequently, it is particularly important to combine spatial imaging with spectral analysis to build an "experimental combat environment" to identify and classify the simulated operational environment and camouflage materials.

In this paper, according to the data's characteristics of hyperspectral image, we collected the hyperspectral image data of simulated camouflage scene. After extracting the ROI and PCA, the classification models were established by combining NB algorithm, KNN algorithm, RF algorithm, and SVM algorithm [8-13]. It is of great significance for the development of techniques of identification and classification based on hyperspectral camouflage to realize the accurate detection and recognition of targets in hyperspectral images by fully mining the deep information of the image.

\section{Materials and Methods}

2.1. Equipment and Principle. We used the Hyperspec VNIR-A model hyperspectral imaging spectrometer produced by the American Headwall Company. Figure 1 shows the hyperspectral imaging system.

As shown in Figure 2, it is a hyperspectral imaging system combining a reflective grating with push-sweep imaging, which is composed of a high-resolution CCD camera, a stepping push-sweep platform, a referenced whiteboard with a calibrated reflectance of above 99\%, a totally reflected grating, and other devices. The hyperspectral camera consists of a photosensitive element, a beam splitter, and a lens and slit.

The spectral test range of the hyperspectral camera used in the experiment is $380-1000 \mathrm{~nm}$, the spectral resolution is $2.5 \mathrm{~nm}$, the number of spectral channels is 923 , the number of spatial channels is 1600 , the width of the slit is $25 \mu \mathrm{m}$, the focal length of the lens is $23 \mathrm{~mm}$, the power of halogen lamp is $150 \mathrm{w}$, and the guide length of the moving push-sweep platform is $250 \mathrm{~mm}$.

When collecting image, the incident slit of the spectrometer is parallel to the spectral axis and a spatial axis. As shown in Figure 3, the moving push-sweep platform captures the information of the whole target by scanning the platform continuously and records the two-dimensional spatial information of the target and the one-dimensional spectral information of the current spatial pixel.

2.2. Sample Preparation. Two samples of simulated backgrounds and three samples of camouflage materials were taken in the darkroom, three hyperspectral images were collected for each type of sample, and each image was repeatedly scanned five times to obtain 75 samples. The simulated desert background is composed of an actual sand table and the simulated jungle background consists of many forest leaves. The three kinds of camouflage materials are desert camouflage netting, jungle camouflage netting, and jungle camouflage clothing. Figure 4 shows true colour images of the samples.
The brightness of the halogen lamp's light source and the focal length of the lens were adjusted to make a clear image, avoiding oversaturation of the image caused by too much light entering the camera to the CCD surface or by uneven distribution of light intensity.

According to the distance from the surface of the object to the slit of the hyperspectral camera and the exposure time, the push-sweep speed of the moving platform is calculated and set to ensure the integrity of the spectral and spatial information of the sample.

2.3. Image Preprocessing. After obtaining the hyperspectral images of operational environments and their corresponding camouflage materials, it is necessary to conduct black-and-white correction by referring to the contrastive method of whiteboard to achieve the inversion of reflectivity of samples, so as to reduce the noise of the CCD sensor's dark current and the influence of changes in light intensity on image signals in the experimental environment. The formula is

$$
R_{\text {norm }}=\frac{R_{\text {raw }}-R_{\text {dark }}}{R_{\text {white }}-R_{\text {dark }}},
$$

where $R_{\text {raw }}$ is the original image, $R_{\text {dark }}$ is the referenced image of full dark acquired after screwing on the lens cover, $R_{\text {white }}$ is the referenced image obtained from a standard whiteboard with a $99 \%$ reflectivity, and $R_{\text {norm }}$ is the corrected spectral image [14].

2.4. Region of Interest Extraction. Because the shape and size of the simulated operational environments and the corresponding camouflage materials were different, some of the surfaces reflect strongly. In order to avoid the influence of spectral information in special areas of some samples, such as some forest leaf samples with surfaces polluted by soil and rain, whose data would have an impact on the overall accuracy of classification, it is necessary to collect image data after cleaning treatment. In the process of ROI extraction, the regions with smooth surface, no abnormal points, regular shape, and clear texture were selected as the research object. According to the principles of selection, this study randomly selected 3 regions with pixel coordinate size of $21 \times 18$ from each sample as ROI for analysis in order to reduce the noise of the experimental data. Part of the results of the ROI is shown in Figure 5.

Each pixel of a hyperspectral image reflects the spectral information of that location. In order to avoid the contingency of each pixel and eliminate the influence of random noise, the pixels in the region of interest and their nearby pixels were averaged as sampling points in the experiment.

2.5. Extraction of Feature Space. Hyperspectral images of the operational environments and their corresponding camouflage materials were acquired, and the image of samples after extracting region of interest records the information of spectral bands of hundreds of dimensions. Yet, the increase 


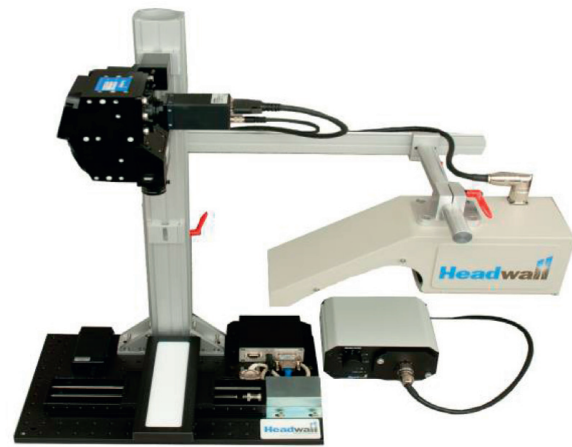

FIGURE 1: Hyperspec VNIR-A model hyperspectral imaging spectrometer produced by American Headwall Company.

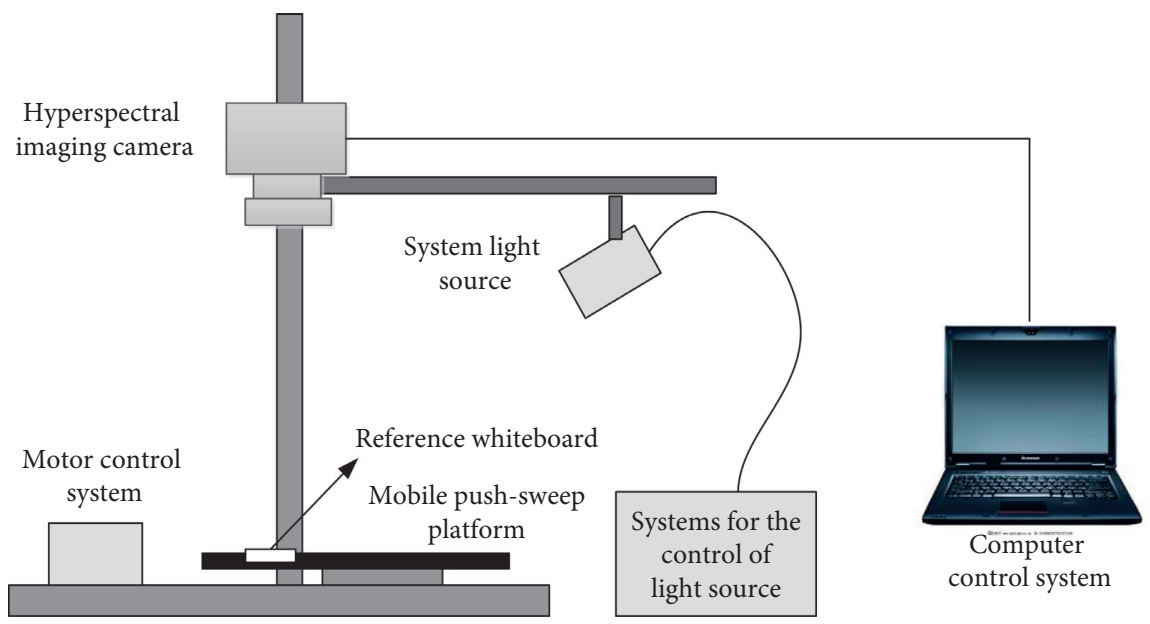

Figure 2: Push-sweep (reflective grating) hyperspectral imaging system.

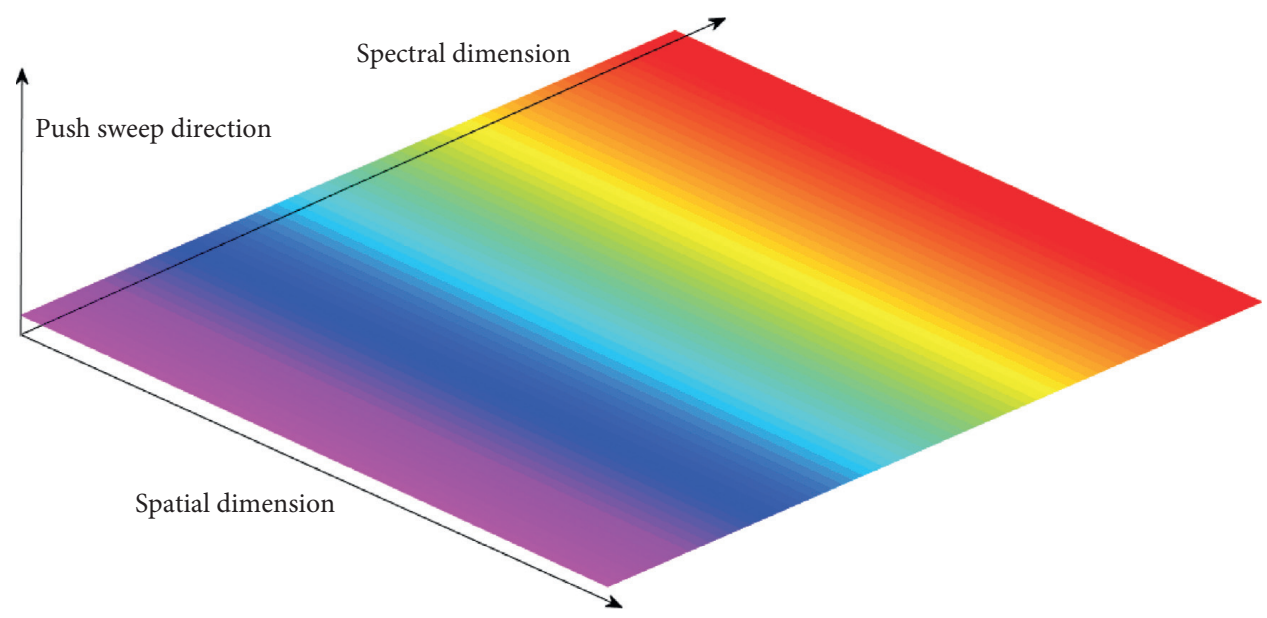

FIGURE 3: Push-sweep imaging schematic.

of band will lead to the redundancy of information and increased complexity of data processing. Principal component analysis uses fewer irrelevant variables to replace the original variables with overlapping information $[13,15]$, which can reduce the data's dimension and ensure the maximum amount of information "not to be lost" $[16,17]$. The specific implementation is as follows:
Set a training set, each sample has $\mathrm{N}$-dimensional features. After centralizing the vectors, the covariance matrix of the new set of vectors is calculated and the corresponding eigenvalues $\lambda_{k}(k=1,2, \ldots, N)$ and eigenvectors are derived. These eigenvalues are arranged from largest to smallest, selecting the first $i$ feature vectors and their corresponding feature vectors to obtain the new features after dimensionality reduction: 


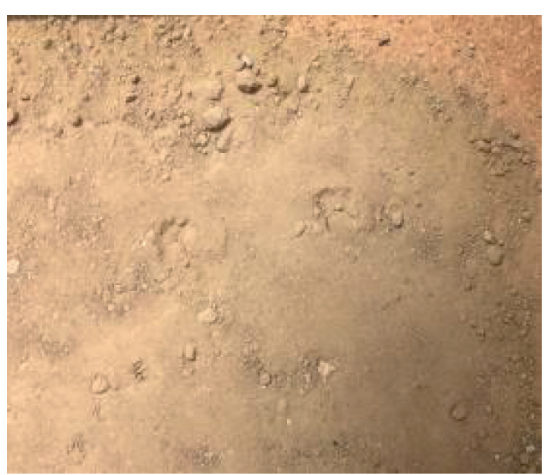

(a)

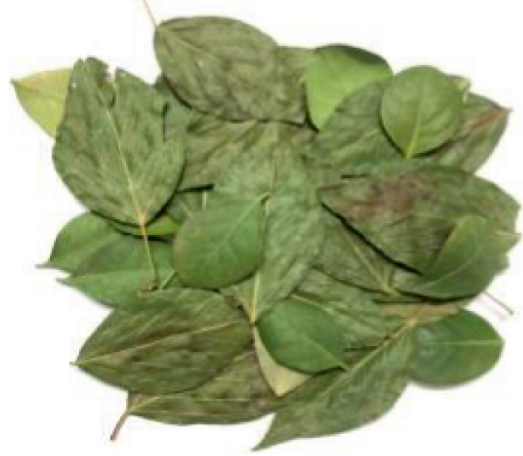

(b)

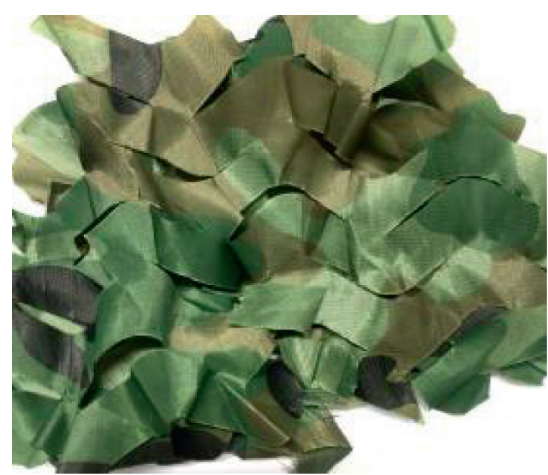

(c)

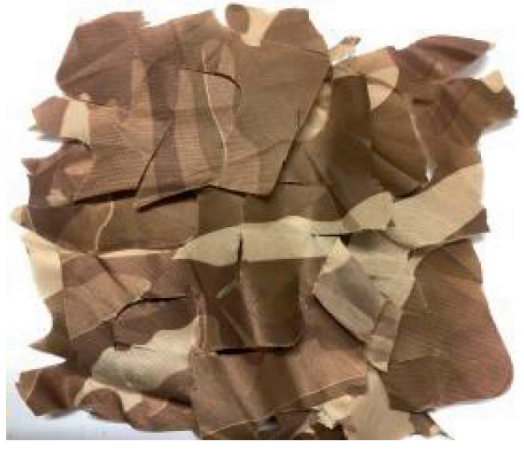

(d)

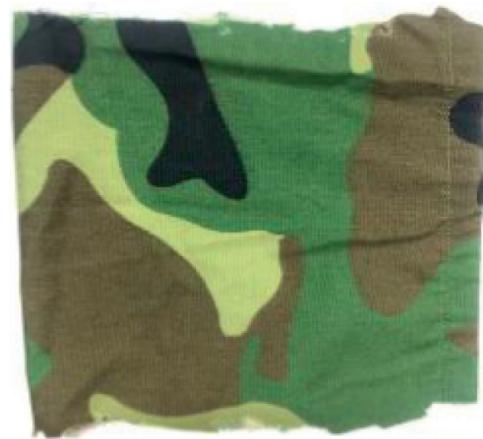

(e)

Figure 4: True colour images of samples. (a) An actual sand table. (b) Forest leaves. (c) Jungle camouflage netting. (d) Desert camouflage netting. (e) Jungle camouflage clothing.

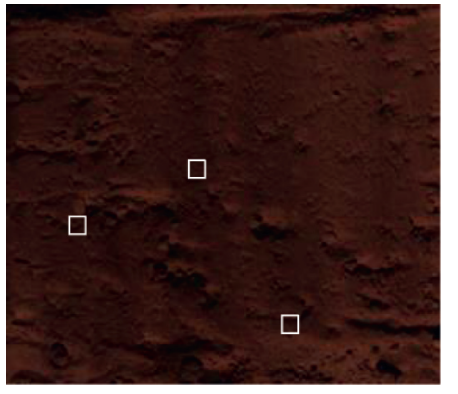

(a1)

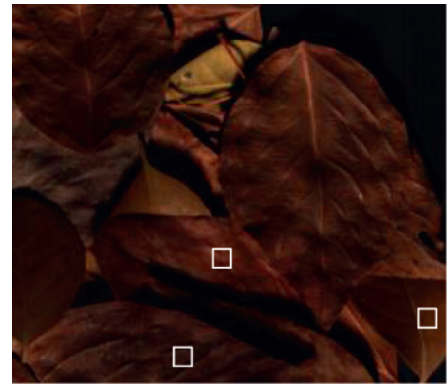

(b1)

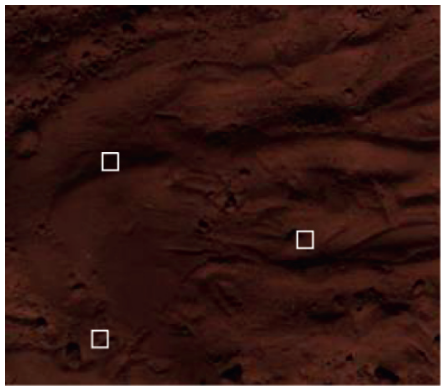

(a2)

(a)

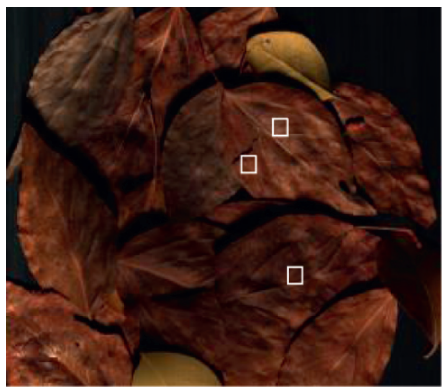

(b2)

(b)

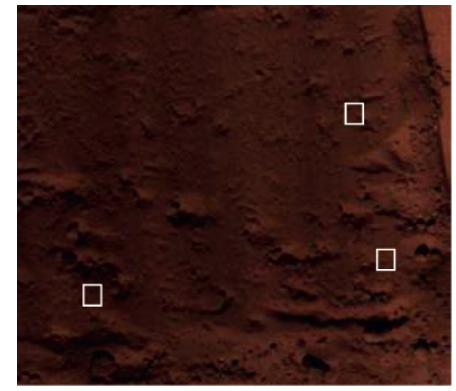

(a3)

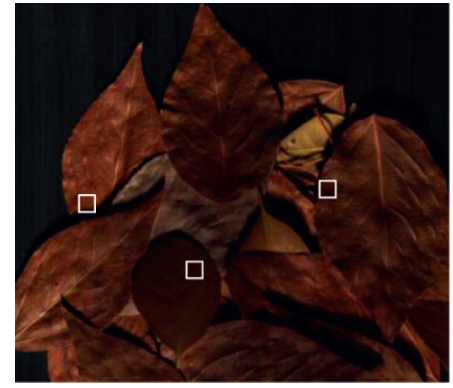

(b3)

Figure 5: Continued. 


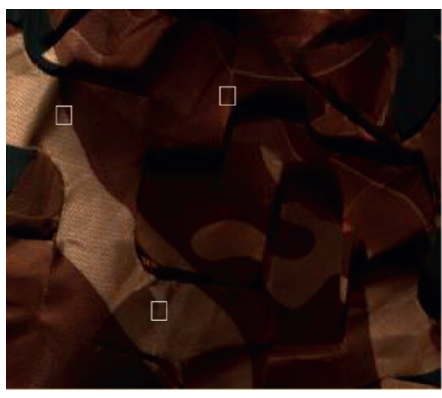

(c1)

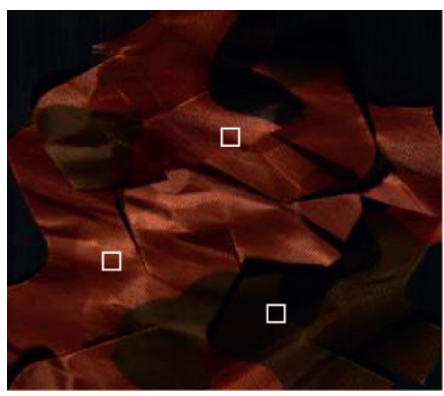

(d1)

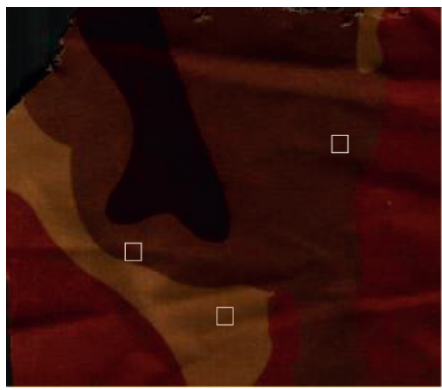

(e1)

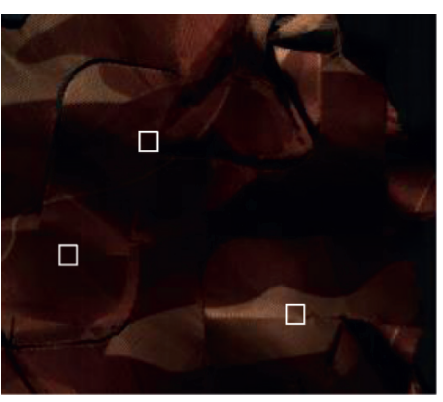

(c2)

(c)

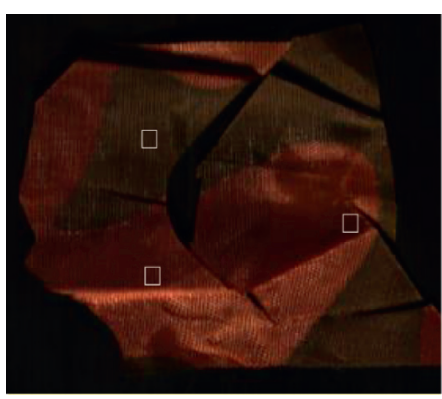

(d2)

(d)

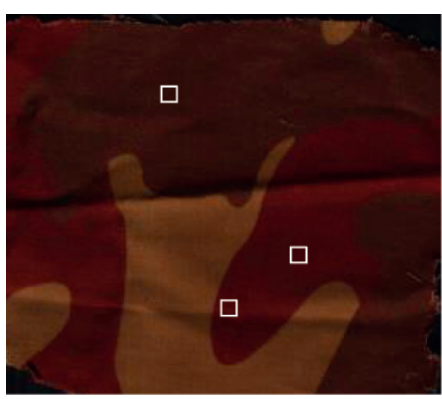

(e2)

(e)

Figure 5: Part of the results of the ROI. (a) Part of the results of ROI in desert background. (b) Part of the results of ROI in jungle background. (c) Part of the results of ROI in desert camouflage netting. (d) Part of the results of ROI in jungle camouflage netting. (e) Part of the results of ROI in jungle camouflage clothing.

$$
a_{i}=\frac{\lambda_{i}}{\sum \lambda_{i}} .
$$

The contribution rate of each principal component is calculated by formula (2). In this study, the cumulative contribution rate of the first three principal components reached $93.57 \%$, with the fourth and subsequent principal components contributing less than 1\%. Figure 6 shows that the first three principal components explained $93.57 \%$ of all information in the original data:

$$
L\left(Z_{i}, X_{m}\right)=\sqrt{\lambda_{i}} a_{i j}
$$

$L\left(Z_{i}, X_{m}\right)$ is the load coefficient of the correlation degree between principal component $Z_{i}$ and original variable $X_{m}$ [18]. The curve of the loading coefficients corresponding to the first three principal components can be obtained by PCA algorithm: the corresponding load factor at the $427.478,449.899,495.359,528.670,560.337$, $585.520,600.497,647.567,686.871,733.175,777.495$, $790.514, \quad 822.793,863.123,895.369, \quad 933.645$, and $972.380 \mathrm{~nm}$ bands had a larger absolute value. Therefore, the feature space corresponding to these 17 wavelengths represented the original information for camouflage recognition and classification.

50 pixels were randomly selected within the image of each sample, while the spectral information of four adjacent pixels was taken for averaging to exclude the effect of random noise. The spectral vector information contained in the 3750 pixels was classified according to categories, and the average spectral curves of the five categories of samples under the extracted 17 wavelengths are shown in Figure 7. 


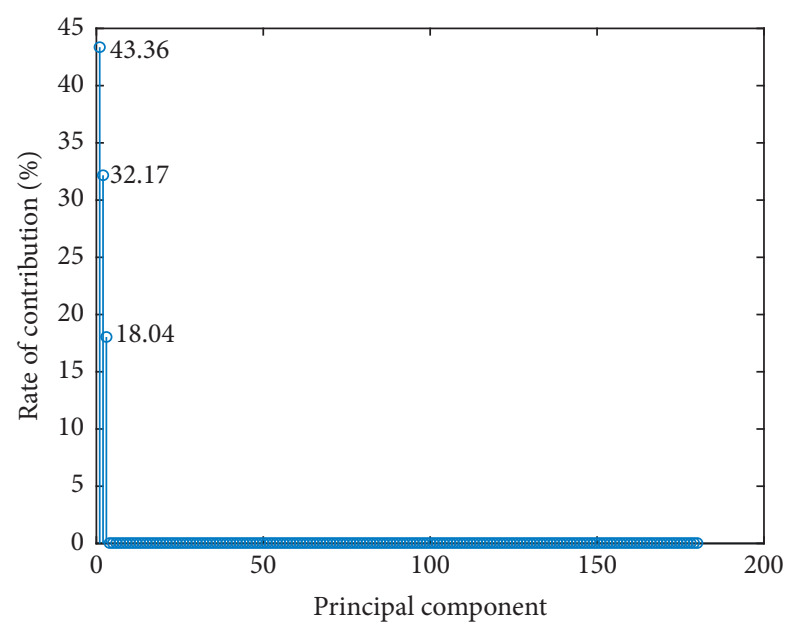

Figure 6: Contribution rate of principal components.

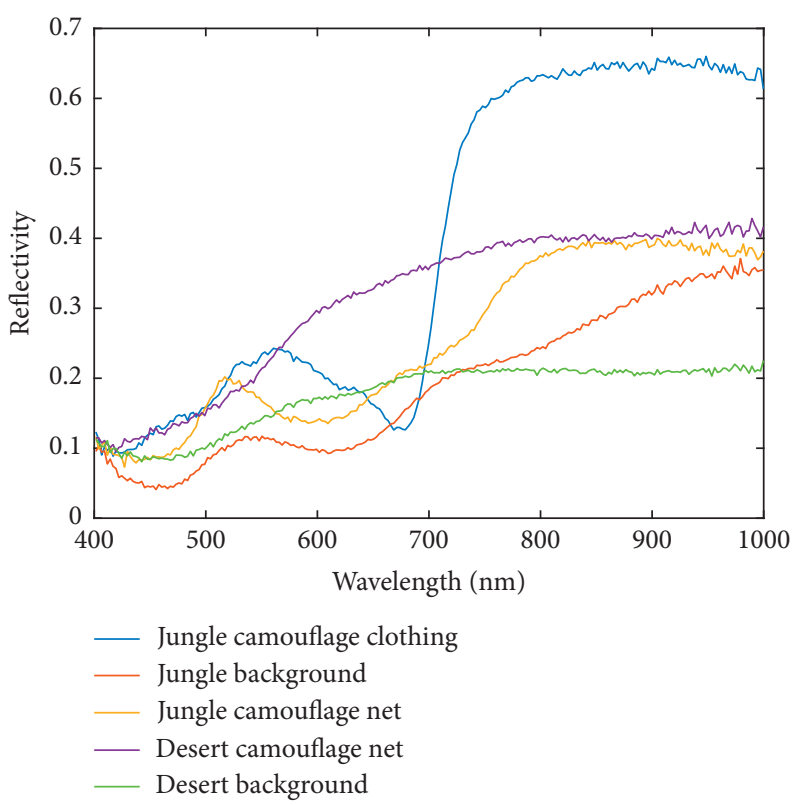

FIGURE 7: Referenced spectral curves of desert background, desert camouflage netting, jungle background, jungle camouflage netting, and jungle camouflage clothing.

\subsection{Classification Models}

2.6.1. The Model of Naive Bayes Algorithm. Naive Bayes algorithm is based on Bayes theorem; the probability of jointed distribution from input to output is learned from a given training set. It is assumed that the data are independent, and the output with the highest posterior probability is found under the learning model [19]. The logic of the algorithm is simple and the output is stable.

2.6.2. The Model of KNN Algorithm. KNN algorithm determines the category of test samples according to the categories of the nearest referenced samples. When the data of the test samples and some training samples are completely matched, the correlation between the samples can be judged by the similarity and classified [20]. The KNN algorithm makes no assumptions about the data, is highly accurate and is insensitive to outliers, making it very suitable for dealing with classification and regression problems[21].

2.6.3. The Model of RF Algorithm. Random forest is an ensemble learning algorithm and a model of decision tree based on bagging framework, which contains multiple decision trees. When a new sample enters the model, each decision tree will produce a result of classification accordingly. Random forest integrates all the voting results and designates the category with the most votes as the final output, that is, the predicted results [22]. Because each tree can be generated independently at the same time, the integrated algorithm is adopted; the accuracy of the integrated algorithm is better than most single algorithms, which makes the results of the whole model have higher accuracy and generalization performance [23]. 
TABLE 1: Classification results of NB model.

\begin{tabular}{lccccccc}
\hline \multirow{2}{*}{ Sample } & \multirow{2}{*}{ Number of tested points } & \multicolumn{3}{c}{ Classification of test samples } & \multicolumn{2}{c}{ Accuracy of classification (\%) } \\
& & 0 & 1 & 2 & 3 & 4 & 70.02 \\
1 & 6804 & 4764 & 567 & 134 & 0 & 1339 & 72.34 \\
2 & 6804 & 718 & 4922 & 340 & 0 & 824 & 73.75 \\
3 & 6804 & 150 & 276 & 5018 & 1322 & 38 & 89.40 \\
4 & 6804 & 0 & 9 & 712 & 6083 & 0 & 72.49 \\
\hline
\end{tabular}

TABLe 2: Classification results of KNN model.

\begin{tabular}{lccccccc}
\hline \multirow{2}{*}{ Sample } & \multirow{3}{*}{ Number of tested points } & \multicolumn{4}{c}{ Classification of test samples } & \multicolumn{2}{c}{ Accuracy of classification (\%) } \\
& & 0 & 1 & 2 & 3 & 4 & 79.66 \\
1 & 6804 & 5420 & 425 & 23 & 0 & 936 & 82.26 \\
2 & 6804 & 679 & 5597 & 64 & 0 & 464 & 81.42 \\
3 & 6804 & 190 & 156 & 5540 & 745 & 173 & 97.78 \\
4 & 6804 & 0 & 2 & 149 & 6653 & 0 & 82.38 \\
\hline
\end{tabular}

TABLE 3: Classification results of RF model.

\begin{tabular}{lccccccc}
\hline \multirow{2}{*}{ Sample } & \multirow{2}{*}{ Number of tested points } & \multicolumn{3}{c}{ Classification of test samples } & \multicolumn{2}{c}{ Accuracy of classification (\%) } \\
& & 0 & 1 & 2 & 3 & 4 & 96.40 \\
1 & 6804 & 6559 & 109 & 13 & 0 & 123 & 97.33 \\
2 & 6804 & 89 & 6622 & 44 & 2 & 47 & 96.81 \\
3 & 6804 & 4 & 17 & 6587 & 189 & 7 & 98.02 \\
4 & 6804 & 0 & 4 & 130 & 6669 & 1 & 97.71 \\
\hline
\end{tabular}

2.6.4. The Model of SVM Algorithm. Support vector machine is a representative supervised statistical learning theory suitable for classification and regression problems, which can prevent underfitting and overfitting very well [24]. Based on the principle of minimizing structural risks, SVM algorithm can avoid the problem of overlearning, and different kernel functions can be used to distinguish the linear and nonlinear data. The samples in this study are nonlinear, so the Gaussian radial basis function (RBF) is chosen as the kernel of SVM mathematical model, which has fast speed of classification and good results.

\section{Results and Discussion}

3.1. Model Comparison. In this section, the Kennard-Stone (K-S) algorithm was applied to divide the samples of region of interest extracted in Section 2.1 [25]. We used the desert background (0), desert camouflage netting (1), jungle background (2), jungle camouflage clothing (3), and jungle camouflage netting (4) in the region of interest of $60 \%$ as the training samples, while the remaining $40 \%$ area was used as the test samples, and each type of test sample contains 6804 tested points. Then, we compared and discriminated with five referenced spectral curves and obtained the results of classification, as shown in Tables 1-4.

$\mathrm{NB}, \mathrm{KNN}, \mathrm{RF}$, and SVM were applied for modeling. The observed values of the correct and incorrect classes of the prediction set could be visually obtained in the table and can be used to calculate corresponding metrics. For the five types of samples, there is easy confusion between the desert background (0), desert camouflage netting (1), and jungle camouflage netting (4) samples.

The results of the classification are shown in Figure 8; when the same method of dimensionality reduction was used, PCA-SVM exhibited the optimal performance for recognition compared to PCA-NB, PCA-KNN, and PCARF.

Comparing the three camouflage materials, jungle camouflage clothing has the highest recognition rate among the four models, reaching $96.30 \%$. The desert camouflage netting is made of the same material as the jungle camouflage netting, which resulted in a less favourable results of the classification than the jungle camouflage clothing, with recognition rates of $87.89 \%$ and $88.14 \%$, respectively.

3.2. Analysis and Discussion. In this study, overall accuracy (OA) and kappa coefficient were used to judge the accuracy of the models of NB, KNN, RF, and SVM.

$\mathrm{OA}$ is the ratio of the number of samples correctly classified by the ROI to the total number of samples within the ROI; the larger it is, the higher the accuracy of classification will be.

Kappa coefficient is an index to measure the accuracy of classification, which represents the proportion of error reduction between classification and completely random classification, and the higher the kappa coefficient, the smaller the difference between the results of the classification and the real reference. Kappa coefficient usually falls between 0 and 1 , which can be divided into five groups to 
TABLE 4: Classification results of SVM model.

\begin{tabular}{|c|c|c|c|c|c|c|c|}
\hline \multirow{2}{*}{ Sample } & \multirow{2}{*}{ Number of tested points } & \multicolumn{5}{|c|}{ Classification of test samples } & \multirow{2}{*}{ Accuracy of classification (\%) } \\
\hline & & 0 & 1 & 2 & 3 & 4 & \\
\hline 0 & 6804 & 6793 & 9 & 2 & 0 & 0 & 99.84 \\
\hline 1 & 6804 & 18 & 6778 & 5 & 0 & 3 & 99.62 \\
\hline 2 & 6804 & 0 & 0 & 6804 & 0 & 0 & 100 \\
\hline 3 & 6804 & 0 & 0 & 0 & 6804 & 0 & 100 \\
\hline 4 & 6804 & 0 & 0 & 0 & 0 & 6804 & 100 \\
\hline
\end{tabular}

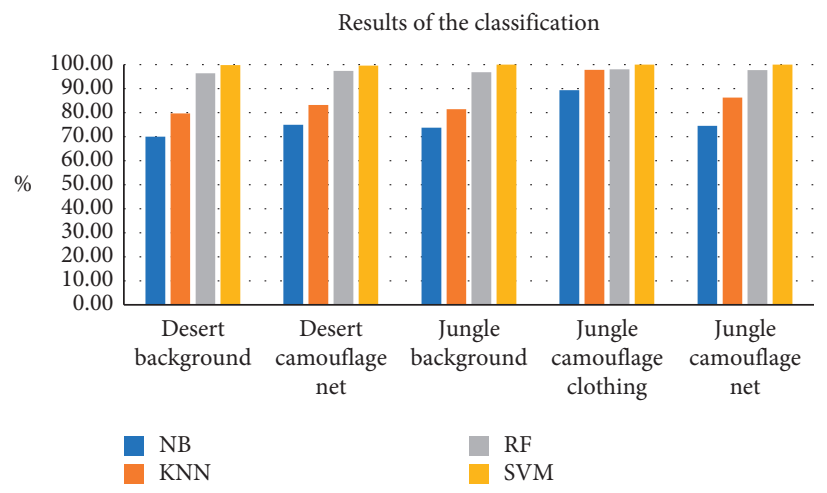

FIgURE 8: Classification results of NB, KNN, RF, and SVM.

TABLE 5: Classification accuracy of four models.

\begin{tabular}{lcccc}
\hline Classification methods & NB & KNN & RF & \\
\hline OA\% & 75.60 & 84.70 & 97.25 & 99.89 \\
Kappa & 0.6950 & 0.8088 & 0.9656 & 0.9986 \\
\hline
\end{tabular}

express different levels of consistency: $0.0-0.20$ (extremely low), 0.21-0.40 (fair), 0.41-0.60 (moderate), 0.61-0.80 (substantial), and $0.80-1$ (almost perfect). The accuracy of classification results is shown in Table 5 .

The NB algorithm assumes that the datasets are independent of each other, but there are some correlations between the operational environments and their camouflage materials, making for poor results of the classification. As a lazy learning method, KNN has a slow prediction time when the number of features is large, which is not an optimal solution in practical application. Accordingly, both RF and SVM can achieve high accuracy and the results of classification are highly consistent with the real results. PCA-RF and PCA-SVM were the optimal models, with both achieving recognition rates of over $96 \%$.

\section{Conclusions}

This study used hyperspectral imaging technology to identify and classify the simulated operational backgrounds and camouflage materials. The methods of establishing and evaluating models are universally valid, providing a new technical route for the effective identification of camouflaged targets in simulated operational environments.

In future research, the model platform can enrich the model by collecting more data, establishing a hyperspectral image database and carrying out continuous optimisation, which will help improve the intelligence and informatization of the future operational environment.

\section{Data Availability}

The data used to support the findings of this study are available from the corresponding author upon request.

\section{Conflicts of Interest}

The authors declare that there are no conflicts of interest regarding the publication of this paper.

\section{Acknowledgments}

This research was funded by the National Natural Science Foundation of China (no. 62005254), Shanxi Province Science Foundation for Youths (no. 201901D211280), and Scientific and Technological Innovation Programs of Higher Education Institutions in Shanxi (no. 2019L0565).

\section{References}

[1] M. Mischiati and P. S. Krishnaprasad, "The dynamics of mutual motion camouflage," Systems \& Control Letters, vol. 61, no. 9, pp. 894-903, 2012.

[2] L. de Filippis, G. Guglieri, and F. Quagliotti, "Path planning strategies for UAVS in 3D environments," Journal of Intelligent \& Robotic Systems, vol. 65, no. 1-4, pp. 247-264, 2012.

[3] Y. Xu and G. Basset, "Virtual motion camouflage based phantom track generation through cooperative electronic combat air vehicles," Automatica, vol. 46, no. 9, pp. 1454-1461, 2010. 
[4] Z. Ji, S. Jia, and L. Shen, "Unsupervised band selection for hyperspectral imagery classification without manual band removal," IEEE Journal of Selected Topics in Applied Earth Observations and Remote Sensing, vol. 5, no. 2, pp. 531-543, 2012.

[5] Y. Zhang, J. Chen, and L. Shen, "Hybrid hierarchical trajectory planning for a fixed-wing UCAV performing air-tosurface multi-target attack," Journal of Systems Engineering and Electronics, vol. 23, no. 4, pp. 256-264, 2012.

[6] C. Zakian, I. Pretty, and R. Ellwood, "Near-infared hyperspectral imaging of teeth for dental caries detection," Journal of Biomedical Optics, vol. 14, no. 6, Article ID 064047, 2009.

[7] R. Hsuan and C. Chein-I, "Automatic spectral target recognition in hyperspectral imagery," IEEE Transactions on Aerospace and Electronic Systems, vol. 39, no. 4, pp. 12321249, 2003.

[8] C. Ke, "Military object detection using multiple information extracted from hyperspectral imagery," in Procedings of the 2017 International Conference on Progress in Informatics and Computing (PIC), pp. 124-128, Nanjing, China, 2017.

[9] G. Notesco, E. Ben Dor, and A. Brook, "Mineral mapping of makhtesh ramon in Israel using hyperspectral remote sensing day and night LWIR images," in Procedings of the 2014 6th Workshop on Hyperspectral Image and Signal Processing: Evolution in Remote Sensing (WHISPERS), pp. 1-4, Lausanne, Switzerland, 2014.

[10] B. Y. Lu, Y. L. Chen, and Y. Y. Li, "The forecast of the preprocessing data with BP neural network and principal component analysis," Science \& Technology Information, vol. 17, pp. 29-30, 2009.

[11] R. A. Poldrack, "Region of interest analysis for fMRI," Social Cognitive and Affective Neuroscience, vol. 2, no. 1, pp. 67-70, 2007.

[12] P. K. Douglas, S. Harris, A. Yuille, and M. S. Cohen, "Performance comparison of machine learning algorithms and number of independent components used in fMRI decoding of belief vs. disbelief," NeuroImage, vol. 56, no. 2, pp. 544-553, 2011.

[13] L.-N. Yang, L. Peng, L.-M. Zhang, L.-l. Zhang, and S.-S. Yang, "A prediction model for population occurrence of paddy stem borer (Scirpophaga incertulas), based on back propagation artificial neural network and principal components analysis," Computers and Electronics in Agriculture, vol. 68, no. 2, pp. 200-206, 2009.

[14] Y.-Q. Zhao and J. Yang, "Hyperspectral image denoising via sparse representation and low-rank constraint," IEEE Transactions on Geoscience and Remote Sensing, vol. 53, no. 1, pp. 296-308, 2015.

[15] Bo Jiang, C. Ding, and J. Tang, "Graph-Laplacian PCA: closed form solution and robustness," in Proceedings of the IEEE Conference on Computer Vision and Pattern Recognition, Port Land, OR, USA, June 2013.

[16] C. Ding, D. Zhou, X. He, and H. Zha, "R 1-PCA: rotational invariant L 1-norm principal component analysis for robust subspace factorization," in Proceedings of the 23rd International Conference on Machine Learning, pp. 281-288, ACM, Pittsburgh, PA, USA, June 2006.

[17] C.-M. Feng, Y.-L. Gao, J.-X. Liu, C.-H. Zheng, and J. Yu, "PCA based on graph Laplacian regularization and $\mathrm{P}$-norm for gene selection and clustering," IEEE Transactions on Nanobioscience, vol. 16, no. 4, pp. 257-265, 2017.

[18] C.-M. Feng, "Joint-norm constraint and graph-Laplacian PCA method for feature extraction," BioMed Research International, vol. 201714 pages, Article ID 5073427, 2017.
[19] J. Cao, R. Panetta, S. Yue, A. Steyaert, M. Young-Bellido, and S. Ahmad, "A naive Bayes model to predict coupling between seven transmembrane domain receptors and G-proteins," Bioinformatics, vol. 19, no. 2, pp. 234-240, 2003.

[20] C. Ji, T. Dong, Y. Li et al., "Inverted grid-based KNN query processing with mapreduce," in Proceedings of the 2012 Seventh ChinaGrid Annual Conference, IEEE, Beijing, China, September 2012.

[21] J. Ma, X. Li, X. Tao, and J. Lu, "Cluster filtered KNN: a WLAN based indoor positioning scheme," in Proceedings of the 2008 International Symposium on a World of Wireless, Mobile and Multimedia Networks (WOWMOM), pp. 1-8, Newport Beach, CA, USA, June 2008.

[22] R. Genuer, J.-M. Poggi, and C. Tuleau-Malot, "Variable selection using random forests," Pattern Recognition Letters, vol. 31, no. 14, pp. 2225-2236, 2010.

[23] B. H. Menze, B. M. Kelm, D. N. Splitthoff, U. Koethe, and F. A. Hamprecht, "On oblique random forests," Machine Learning and Knowledge Discovery in Databases, vol. 6912, pp. 453-469, 2011.

[24] G. Zhu and D. G. Blumberg, "Classification using ASTER data and SVM algorithms: the case study of Beer Sheva, Israel," Remote Sensing of Environment, vol. 80, no. 2, pp. 233-240, 2002.

[25] Y. Chen, R. Zhao, and T. Qi, “A strategy for constructing a calibration set for a model for estimating total nitrogen in rice soils combining spectral transformations and the KennardStone algorithm," Spectroscopy and Spectral Analysis, vol. 37, no. 7, pp. 2133-2139, 2017. 